\title{
Stray Flux-Based Rotation Angle Measurement for Bearing Fault Diagnosis in Variable-Speed BLDC Motors
}

\author{
Xiaoxian Wang, Siliang Lu, Senior Member, IEEE, Wenping Cao, Senior Member, IEEE, \\ Min Xia, Kang Chen, Jianming Ding, and Shiwu Zhang, Member, IEEE
}

\begin{abstract}
Angle of rotation is a key parameter in motor fault diagnosis under varying speed conditions, and is usually measured by an optical encoder. However, the use of encoders is intrusive and in many scenarios its signal is difficult to access due to technical or commercial reasons. In this study, a novel rotation angle measurement method based on stray flux analysis is proposed and applied to bearing fault diagnosis of brushless direct-current (BLDC) motors. The measurement accuracy of the proposed method is comparable to that from an encoder. The developed method is flexible, noninvasive, and nondestructive. It is easy to implement and eliminates the need for long cables and access of the motor control system. The proposed method can be extended to the diagnosis of motor electrical and drive faults. If implemented with an Internet of Things (IoT) or a hand-held device, it can further improve the reliability of sensorless motor drive systems in industrial automation so as to meet Industry 4.0 requirements.
\end{abstract}

Index terms-Bearing fault, BLDC motors, fault diagnosis, rotation angle measurement, stray flux, vibration signal analysis

\section{INTRODUCTION}

Brushless direct current (BLDC) motors are a type of common pivotal actuators used in the field of industrial

This work was supported in part by the National Natural Science Foundation of China (No. 52075002), and the Open Project Program of the Traction Power State Key Laboratory of Southwest Jiaotong University (TPL1905). (corresponding author: Siliang $\mathrm{Lu}$ and Shiwu Zhang)

Xiaoxian Wang is with the Traction Power State Key Laboratory, Southwest Jiaotong University, Chengdu 610031, China, and the College of Electronics and Information Engineering, Anhui University, Hefei 230601, China, and the Department of Precision Machinery and Precision Instrumentation, University of Science and Technology of China, Hefei 230027, China.

Siliang Lu is with the Traction Power State Key Laboratory, Southwest Jiaotong University, Chengdu 610031, China, and the College of Electrical Engineering and Automation, Anhui University, Hefei 230601, China. (email: lusliang@mail.ustc.edu.cn)

Wenping Cao is with the School of Engineering and Applied Science, Aston University, Birmingham B4 7ET, U.K., and the College of Electrical Engineering and Automation, Anhui University, Hefei 230601, China.

Min Xia is with the Department of Engineering, Lancaster University, Lancaster, LA1 4YW, U.K.

Kang Chen is with the National Engineering Laboratory of Energy-Saving Motor and Control Technology, College of Electrical Engineering and Automation, Anhui University, Hefei 230601, China.

Jianming Ding is with the Traction Power State Key Laboratory, Southwest Jiaotong University, Chengdu 610031, China.

Shiwu Zhang is with the CAS Key Laboratory of Mechanical Behavior and Design of Materials, Department of Precision Machinery and Precision Instrumentation, University of Science and Technology of China, Hefei 230027 China. (email: swzhang@ustc.edu.cn) automation. Early fault diagnosis is of critical importance as it can extend the healthy operation of motors and reduce the likelihood of severe accidents. Typical motor failures are classified into three types: electrical, magnetic, and mechanical failures. The electrical faults include winding interturn short-circuit faults, winding open-circuit faults, winding resistance imbalance, and broken rotor bars [1, 2]. The magnetic faults include magnet damage and demagnetization, and iron core defects [3]. The mechanical faults involve bearing faults and rotor eccentricity $[4,5]$. Typical mechanical faults can directly lead to a breakdown of the motor drives while electrical faults take a longer time to develop from a minor to a severe fault. Therefore, this study focuses on bearing faults, which account for about $45 \%$ of the total motor faults [6].

The motor bearings are usually subjected to cyclic loads at axial direction, and the alternating stress can lead to fatigue after a certain of cycle times. The fatigue further leads to crack, pitting, and scratch. The incipient failures will expand to large-size ones and finally to severe failures such as broken cage and rollers, wearing of outer and inner raceways. The original manufacture defects or improper operation (misalignment or overloading) can accelerate the degradation of the bearing [7].

Bearing faults produce vibration and noise that can be detected externally. In industry, vibration signal analysis is one of the most effective methods. When a motor operates at a constant speed, the fault signatures appear periodically in the acquired vibration signal. Waveform or spectral analysis methods can be used to estimate the intervals or frequencies of the periodic features. However, if the shaft speed varies with time, the fault features become non-stationary, and the traditional spectral analysis becomes less effective. Under this condition, an order analysis technique can be used to process the time-varying signals in the angular domain [8]. In this case, the rotation angle information is required to aid in the analysis. This can be obtained from an encoder if it is installed in the motor shaft. However, many industrial motors are based on sensorless control where an encoder is not employed $[9,10]$.

Thus, the rotating angle must be estimated from other signals, such as video stream, motor current, and vibration signal [11]. For instance, a vision-based measurement system that uses a linearly varying density fringe pattern was designed for speed monitoring [12]. A current-aided order analysis method was developed for bearing fault diagnosis [13]. A rolling element 
$>$ IEEE Transactions on Energy Conversion<

bearing fault diagnosis method based on fault characteristic order (FCO) analysis was proposed in [14], in which the rotation angle is estimated from the vibration signal. More details about rotation angle or speed measurement methods without using a tachometer can be found in a review article [8].

Whilst existing angle measurement methods are effective in different mechanisms, they have their limitations. For instance, video signals can provide the rotation angle but require the use of an expensive high-speed camera [15]. The measurement accuracy of the camera is affected by several factors, such as illumination intensity and surface texture. Similarly, vibration signals are rich and easy to capture but its measurement accuracy is affected by the surrounding environment [13]. Machine winding currents can be presented with some bearing fault signatures, but these are transmitted through the main flux and stator windings. Any disturbance and interference can impact on the measurement accuracy.

In this study, a method based on stray magnetic signals is proposed for motor rotation angle measurement. The advantages of the proposed method are evaluated in a BLDC motor servo drive unit in industrial automation. As shown in Fig. 1, this unit consists of a BLDC servo motor, a power supply, a controller, current, flux and vibration sensors, and connection cables. The motor rotation angle can be obtained from an encoder, a current probe, and a stray flux sensor for comparison purposes. The measured data are conditioned through a data acquisition system (DAS) which is connected to a PC for analysis. The interfaces of the current sensor and the encoder are located on the electrical cabinet side, and no connector is exposed on the actuator side. For this reason, several long cables should be wired between the motor and the power supply to obtain the angle signal.

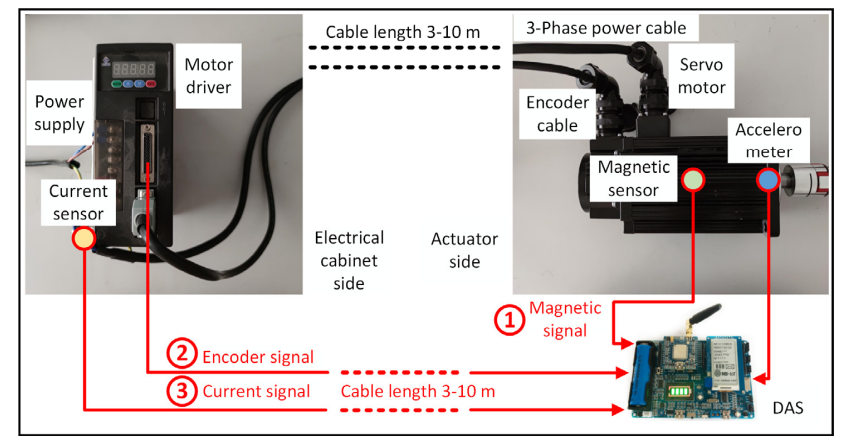

Fig. 1. Different rotation angle measurement methods for the fault diagnosis of a typical servo driving unit under variable speed conditions.

However, wiring long signal cables is undesirable in industrial applications. Mutual interference between cables may occur and the impedance of cables may give rise to measurement uncertainties. By contrast, magnetic sensors can capture the stray flux leaking from the main flux and they can be installed on the motor housing [16]. A relatively short signal cable is used as the magnetic sensor is close to the DAS. In addition, the DAS, magnetic sensor, and accelerometer can be integrated into one unit in Internet of Things (IoT) applications or a hand-held instrument to eliminate the use of connection cables and improve flexibility.
Although magnetic sensors are promising tools for measuring the rotation angle, the stray magnetic signals are nonlinear and weak. The technical challenge lies in the measurement accuracy [17]. In this study, a series of algorithms are proposed to achieve high-accuracy measurements from stray magnetic signals. The novelty and contributions of this study are summarized as follows. First, this study is the first to propose a stray flux-based BLDC motor rotation angle measurement. Second, the flux signal is processed adaptively, and the accuracy of the measured angle curve is comparable to that obtained from optical encoders. Lastly, this study introduces a new approach for motor fault diagnosis under varying speed conditions which eliminates long cable wirings and enables IoT and hand-held instrument applications.

The remainder of the paper is organized as follows. Section II introduces the principle of rotation angle measurements from stray flux signals. Section III presents the new algorithms for angle measurements. Section IV presents the experimental setup. Section V evaluates the performance of the motor rotation angle measurement and fault diagnosis. Section VI verifies the robustness of the proposed method. Section VII discusses the proposed method in practical applications. Section VIII provides the conclusions and further work.

\section{Principle of Rotation Angle Measurements from STRAY MAGNETIC SIGNALS}

The BLDC motor is a permanent magnet synchronous motor energized by a three-phase power converter with trapezoidal currents. The cross-sectional view of the BLDC motor is shown in Fig. 2. The motor stator consists of six salient poles wrapped with three-phase coil windings, whereas the motor rotor comprises two pairs of permanent magnets mounted on the rotor surface. The motor driver energizes the windings with the sequences as: 1) $\mathrm{A}+\mathrm{B}-$, 2) $\mathrm{C}+\mathrm{B}-$, 3) $\mathrm{C}+\mathrm{A}-$, 4) $\mathrm{B}+\mathrm{A}-$, 5) $\mathrm{B}+\mathrm{C}-$, and 6) $\mathrm{A}+\mathrm{C}-$. The stator rotational magnetic field pulls the rotor to rotate synchronously [18].

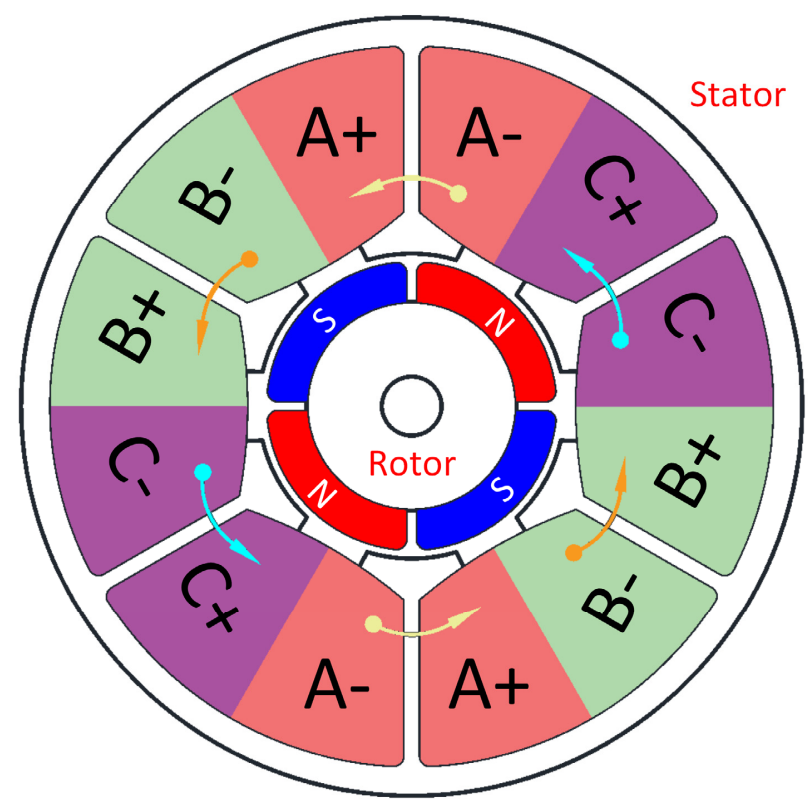

Fig. 2. Cross-sectional view of the BLDC motor. 
$>$ IEEE Transactions on Energy Conversion<

The energy transfers from the stator to the rotor through the air gap. As shown in Fig. 3(a), part of the air-gap flux $\Phi_{a}$ (denoted as $\Phi_{l}$ ) leaks to the outside of the motor housing. This phenomenon can be mathematically expressed as [19]:

$$
\Phi_{l}=p \Phi_{a}
$$

where $p$ is the ratio of flux leakage. The leakage flux density is affected by the structure and material of the iron laminations, reluctance, and position of the magnetic sensor installed in the motor housing [19-22].

In this study, the rotation angle is measured from the magnitude fluctuation of the stray flux density. As this is a synchronous motor, the stray flux changes synchronously with the main flux. The speed of the mechanical shaft can be obtained as:

$$
f_{r}=\frac{f_{s f d}}{n p},
$$

where $f_{s f d}$ is the frequency of the stray flux and $n p$ is the number of motor pole pairs. Fig. 3(b) is an illustration of the waveforms of the air-gap flux $\Phi_{a}$ and leakage flux $\Phi_{l}$ for a motor with $n p=$ 2. A full rotation cycle of the mechanical rotor corresponds to two full cycles of the flux signal. In addition, flux $\Phi_{l}$ is an attenuated version of the flux $\Phi_{a}$, and their period and frequency are strictly equal. Therefore, the rotation angle of the rotor can be measured from the stray flux signal. In this study, the ratio of flux leakage $p$ does not need to know for angle measurement.
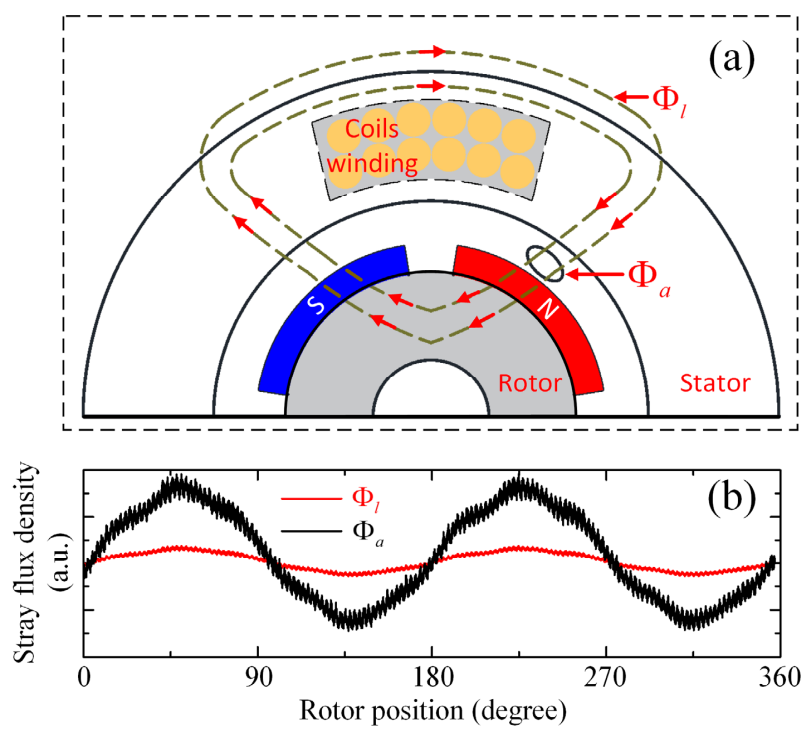

Fig. 3. (a) Illustration of the air-gap flux and leakage flux and (b) their waveforms.

\section{Rotation Angle Measurement And Fault Diagnosis}

In practice, the magnetic signal is impaired by the noise interference. Therefore, a digital signal processing method is proposed to analyze the magnetic signal to obtain an accurate rotation angle measurement. The motor fault diagnosis based on order analysis can be realized on the basis of the measured rotation angle. The method includes magnetic signal time-frequency analysis (TFA) and instantaneous frequency
(IF) extraction, rotation angle calculation, and vibration signal resampling for fault diagnosis.

\section{A. TFA and IF Extraction}

Considering that the time-varying magnetic signal is non-stationary, TFA is used to extract the dominant IF component that is highly related to motor rotation. Many TFA methods, including short-time Fourier transform, Wigner-Viller distribution, and wavelet transform, are studied to analyze vibration signals $[23,24]$. This study adopts the wavelet synchrosqueezed transform (WST) for the TFA because of its high accuracy in IF estimation and extraction [25, 26]. A continuous magnetic signal with $K$ components can be expressed as:

$$
M(t)=\sum_{j=1}^{K} A_{j}(t) \cos \omega_{j}(t)+n(t),
$$

where $A_{j}(t)$ and $\omega_{j}(t)$ are the instantaneous amplitude (IA) and IF of the $j$ th component of the magnetic signal, respectively, $n(t)$ is the noise introduced during the measurement, and $M(t)$ is a time-varying signal. WST extracts and reconstructs the IAs and IFs of the components.

Continuous wavelet transform is applied to $M(t)$ to estimate the IFs as:

$$
W_{M}(a, b)=a^{-1 / 2} \int M(t) \psi^{*}\left(\frac{t-b}{a}\right) d t,
$$

where $a$ and $b$ are the scale and shift coefficients of the wavelets, respectively, $\psi(\cdot)$ is the mother wavelet, and $\psi^{*}(\cdot)$ is its conjugation. The IF for any $W_{M}(a, b) \neq 0$ can be calculated as:

$$
f_{M}(a, b)=\frac{-i \partial\left(W_{M}(a, b)\right)}{W_{M}(a, b) \partial b} .
$$

where $i$ denotes the imaginary unit and it is induced from the cosine components in $M(t)$ according to the Euler's formula, i.e., $\cos \omega_{j}(t)=\left[\exp \left(i \omega_{j}(t)\right)+\exp \left(-i \omega_{j}(t)\right)\right] / 2$. The detailed derivations can be referred to Ref. [25].

Eq. (5) establishes a map from $(b, a)$ to $\left(b, f_{M}(a, b)\right)$. WST then redistributes the energy on the time-scale plane and converts it on the time-frequency plane as [27]:

$$
T_{M}(f, b)=\int_{\left\{a: W_{M}(a, b) \neq 0\right\}} W_{M}(a, b) a^{-3 / 2} \frac{1}{\delta} h\left(\frac{f-f_{M}(a, b)}{\delta}\right) d a,
$$

where $T_{M}(f, b)$ is a function with respect to frequency $f, W_{M}(a, b)$ and $f_{M}(a, b)$ are the functions with respect to $a$ and $b, \delta$ is the accuracy, and $h(\cdot) \in C_{0}^{\infty}$ is a smooth function that satisfies $\|h\|_{L^{1}}=1$. The practical magnetic signal $M[n](n=1,2, \ldots, N$, where $N$ is the signal length) is discrete. The discrete form of Eq. (6) can be written as:

$$
T_{M}\left[f_{l}, b_{n}\right]=\sum_{a_{j}:\left\{f\left(a_{j}, b_{n}\right)-f_{l} \mid \leq \Delta f / 2\right.} W_{M}\left[a_{j}, b_{n}\right] a_{j}^{-3 / 2}(\Delta a)_{j},
$$

where $a_{j}$ and $b_{n}$ are the discrete forms of the wavelet coefficients, $f_{l}$ is the $l$ th discrete angular frequency $\left(\Delta f=f_{l}-f_{l-1}\right)$, and $(\Delta a)_{j}=a_{j}-a_{j-1}$. Considering that the noise is inevitable in a discrete signal, a hard threshold $\lambda$ is introduced to smooth the signal component during computation. Namely, if the value of signal point is smaller or equal to $\lambda$, this point is disregarded. The detailed discussion of the threshold value can be referred in 
$>$ IEEE Transactions on Energy Conversion<

[27].

After TFA, a ridge extraction algorithm is used to obtain the IFs. The locations of the energy peaks in the time axis of the time-frequency plane are denoted as $m_{m}\left[b_{n}\right]$, and the magnitudes of the peak points are denoted as $U_{m}\left[b_{n}\right](m=1$, $\left.2, \ldots, N_{p}\left[b_{n}\right]\right)$. The amplitudes of the ridges are obtained as:

$$
U_{m}\left[b_{n}\right]=\left|y_{m}\left[b_{n}\right]\right|=\left|\sum_{f_{-}^{(m)}\left(b_{n}\right)}^{f_{f}^{(m)}\left(b_{n}\right)} T_{M}\left[f_{l}, b_{n}\right] \Delta f\right|,
$$

where $\left(f_{+}^{(\mathrm{m})}\left[b_{n}\right], f_{-}^{(\mathrm{m})}\left[b_{n}\right]\right)$ is the widest region of the nonzero amplitudes decomposed by WST at time instance $b_{n}$. The frequencies of the ridges are determined as:

$$
m_{m}\left[b_{n}\right] \equiv \operatorname{Real}\left[\left[y_{m}\left[b_{n}\right]\right]^{-1} \sum_{f_{-}^{(m)}\left(b_{n}\right)}^{f^{(m)}\left(b_{n}\right)} f_{l} T_{m}\left[f_{l}, b_{n}\right] \Delta f\right],
$$

where Real[] represents the real part of a complex variable.

The multiple IFs of the magnetic signal are extracted by applying WST and ridge extraction. In accordance with the rotation angle measurement principle in Sec. II, the IF with the highest energy is selected to reconstruct the rotation component without heavy noise interference. The discrete IF and IA curves are denoted as $I F[n]$ and $I A[n](i=1,2, \ldots, N)$, respectively.

\section{B. Rotation Angle Calculations}

The BLDC motor is fed with trapezoidal currents, which can be corrupted by electrical noise. TFA and IF extraction algorithms are used to extract the IF of the signal component relative to the motor rotation. The quadrature parts of the rotation signal component are expressed as $x_{r}[n]$ and $y_{r}[n]$, and this component is reconstructed as:

$$
S_{r}[n]=x_{r}[n] \times \operatorname{cosm}[n]+y_{r}[n] \times \operatorname{sinm}[n], n=1, \ldots, N,
$$

where $\operatorname{cosm}[n]$ and $\operatorname{sinm}[n]$ are expressed as:

$$
\left\{\begin{array}{l}
\operatorname{cosm}[n]=\cos (2 \pi(\operatorname{cumtrapz}(t[n], \operatorname{IF}[n]))) \\
\operatorname{sinm}[n]=\sin (2 \pi(\operatorname{cumtrapz}(t[n], I F[n])))
\end{array} n=1, \ldots, N .\right.
$$

In Eq. (11), $t[n]$ is the synchronous sampling instant sequence of the magnetic and vibration signals and cumtrapz() is a MATLAB function used for cumulative trapezoidal numerical integration. In practice, to improve the computation efficiency, the magnetic signal can be firstly downsampled, and then processed by the WST. Finally, the length of the reconstructed signal is extended to that of the original magnetic signal by using numerical interpolation.

In accordance with the principle of order analysis, the motor rotation angle with respect to time is calculated to resample the vibration signal. The phase of the reconstructed magnetic signal is obtained as:

$$
\operatorname{Pm}[n]=\text { unwrap }\left\{\arctan \left(\frac{\operatorname{Imag}\left(H S_{r}[n]\right)}{\operatorname{Real}\left(H S_{r}[n]\right)}\right)\right\}, n=1,2, \ldots, N,
$$

where $H S_{r}[n]$ is the analytic signal of $S_{r}[n]$ obtained using Hilbert transform, Imag() represents the image part of the signal, and unwrap () is a MATLAB function used to unwrap the signal to obtain a continuous and smooth phase curve.

The mechanical rotation angle of the motor shaft can be calculated as:

$$
R a_{m}[n]=\frac{180^{\circ}}{\pi \times n p} \times(\operatorname{Pm}[n]-P m[1]), n=1,2, \ldots, N .
$$

By introducing the term $-P m[1]$, the initial angle of $R a_{m}[n]$ is set to 0 . Note that the initial angle 0 is a relative angle to represent the start point of the signal segment, and it is independent of the absolute angle of the motor rotor. In other words, the method begins to calculate the angle increment from the first point of the sampled signal. The successive motor rotation angle can be measured adaptively from the noisy magnetic signal by using the above algorithms.

\section{Order Analysis and Fault Diagnosis}

Order analysis involves a numerical interpolation on the time-domain vibration signal in accordance with the vector of the rotation angle. Through this analysis, the dispersive energy of the fault characteristic frequency (FCF) will concentrate to the FCO, which is a beneficial phenomenon for fault identification. The vibration signal synchronously sampled with the magnetic signal is denoted as $\operatorname{Vib}[n](n=1,2, \ldots, N)$. The signal is resampled as:

$$
R V i b[n]=\operatorname{resample}\left(\operatorname{Vib}[n], \operatorname{Ra}_{m}[n]\right),
$$

where resample() is a MATLAB function for signal resampling. The resampled vibration signal, which is still a modulated signal, is demodulated as:

$$
\operatorname{Dem}[n]=\sqrt{(\operatorname{Imag}(H R V i b[n]))^{2}+(\operatorname{Real}(H R V i b[n]))^{2}},
$$

where $H R V i b[n]$ is the analytic signal of $R V i b[n]$ obtained through Hilbert transform. The spectrum of $\operatorname{Dem}[n]$ is computed using fast Fourier transform, and fault diagnosis is conducted by comparing the fault indicator in the spectrum with the theoretical one.

\section{Algorithm Flowchart}

The flowchart of the algorithm of the proposed method is shown in Fig. 4. The left panel shows the flow for measuring the rotation angle from the magnetic signal acquired using a Hall sensor, whereas the right one displays the flow for motor bearing fault diagnosis based on the analysis of the vibration signal acquired using an accelerometer.

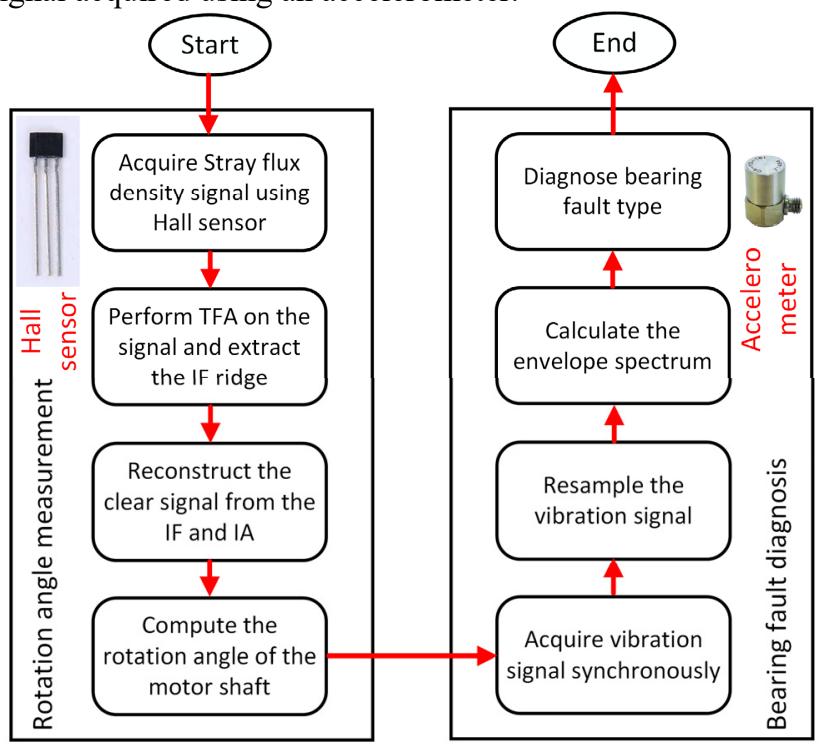

Fig. 4. Flowchart of the algorithm of the proposed method. 
$>$ IEEE Transactions on Energy Conversion<

\section{EXPERIMENTAL SETUP}

The effectiveness of the proposed rotation measurement method is verified through an experiment (Fig. 5). The parameters of the BLDC motor used in the rotation angle measurement are listed in Table I. The test motor drives an AC generator via a mechanical coupling, and the generator connects to an AC-DC converter and then to a DC electrical load (DL3021, Rigol, Inc.). The rotation speed is varied using a speed controller. A Hall sensor (AH49E, BCD Semiconductor Manufacturing Limited) with a typical sensitivity of 1.6 $\mathrm{mV} /$ Gauss is installed at the center of the side face of the motor housing to measure the stray flux density. An encoder (ZSP3806, TOONE, Inc.) with a resolution of 500 pulses per revolution is installed at the motor shaft to evaluate the measurement accuracy of the proposed method. In addition, a current probe (80i-110s, Fluke, Inc.) with a sensitivity of 100 $\mathrm{mV} / \mathrm{A}$ is used to acquire the winding current signal for method comparison. An accelerometer (CA-YD-1182, SINOCERA, Inc.) with a sensitivity of $100 \mathrm{mV} / \mathrm{g}$ is installed at the motor housing to acquire the bearing vibration signal. The magnetic, vibration, encoder, and current signals are acquired using a DAS (USB4432, NI, Inc.) with a sampling frequency of 51.2 $\mathrm{kHz}$ and a sampling duration of $4 \mathrm{~s}$.

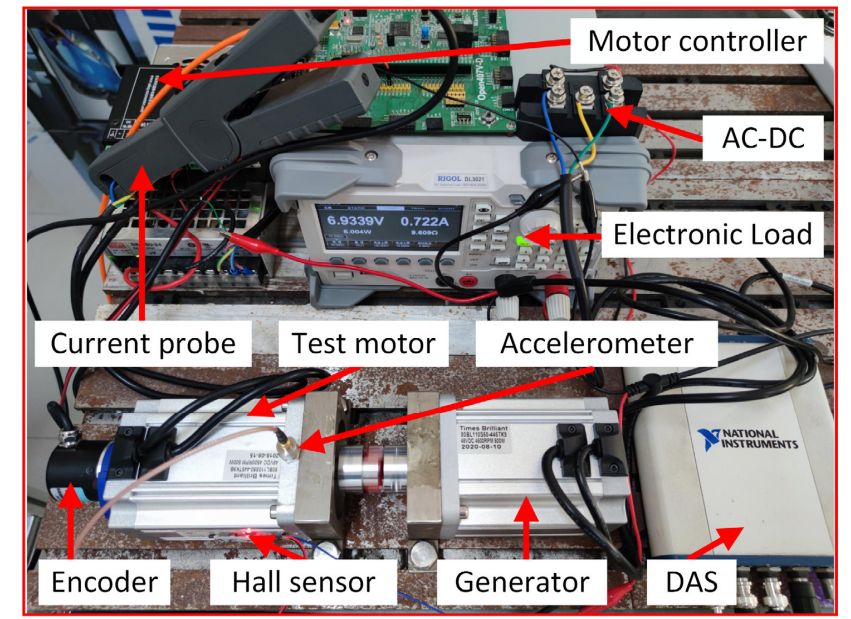

Fig. 5. Experimental setup.

TABLE I

BLDC MOTOR PARAMETERS

\begin{tabular}{ccccccc}
\hline \hline Type & $\begin{array}{c}\text { Rated } \\
\text { Voltage }\end{array}$ & $\begin{array}{c}\text { Rated } \\
\text { Torque }\end{array}$ & $\begin{array}{c}\text { Rated } \\
\text { Power }\end{array}$ & $\begin{array}{c}\text { No. of } \\
\text { Pole } \\
\text { Pairs }\end{array}$ & $\begin{array}{c}\text { No. of } \\
\text { Slots }\end{array}$ & $\begin{array}{c}\text { No. of } \\
\text { Phases }\end{array}$ \\
\hline 80 BL110 & 48 VDC & $1.6 \mathrm{Nm}$ & $500 \mathrm{~W}$ & 2 & 6 & 3 \\
\hline \hline
\end{tabular}

Two deep groove ball bearings with outer and inner raceway faults are separately installed at the side of the output shaft. The fault with a size of $1 \mathrm{~mm} \times 1 \mathrm{~mm}$ (depth $\times$ width) is set through electrical discharge machining. When the bearing rotates, the rolling elements roll over the outer or inner raceway defects and generate the impulses. The period or frequency of the occurrence of the impulses is determined via the bearing's geometric and the rotational speed. Hence, the bearing fault type can be diagnosed through the spectral analysis of the vibration signal. The bearing type and parameters are summarized in Table II. The FCO is the normalized FCF with respect to rotation frequency. The FCO for the outer and inner raceway fault are respectively calculated as [14]:

$$
\begin{aligned}
& F C O_{O}=\frac{N R}{2}\left(1-\frac{d_{1}}{d_{2}} \cos \gamma\right), \\
& F C O_{I}=\frac{N R}{2}\left(1+\frac{d_{1}}{d_{2}} \cos \gamma\right),
\end{aligned}
$$

where $N R$ is the number of rolling elements, $d_{1}$ is the diameter of the rolling element, $d_{2}$ is the pitch diameter, and $\gamma$ is the contact angle. In the test bearing (type 6002), $\gamma$ is $0^{\circ} . F C O_{O}$ and $F C O_{I}$ are calculated based on the bearing parameters as shown in Table II.

TABLE II

BEARING PARAMETERS

\begin{tabular}{cccccc}
\hline \hline Type & $\begin{array}{c}\text { Outer } \\
\text { Diameter } \\
(\mathrm{mm})\end{array}$ & $\begin{array}{c}\text { Inner } \\
\text { Diameter } \\
(\mathrm{mm})\end{array}$ & $\begin{array}{c}\text { Number of } \\
\text { Rolling } \\
\text { Elements }\end{array}$ & FCOO & $F C O_{I}$ \\
\hline 6002 & 32 & 15 & 9 & 3.59 & 5.41 \\
\hline \hline
\end{tabular}

\section{Performances of Motor Rotation Angle MEASUREMENT AND FAULT DiagNOSIS}

In this section, the fault diagnosis performance of the proposed motor rotation angle measurement method is evaluated.

\section{A. Diagnosis of Bearing with Outer Raceway Fault}

The bearing with outer raceway fault is tested firstly. The BLDC motor is configured to spin at variable speed, and the duration of the acquired stray magnetic signal is $4 \mathrm{~s}$ (Fig. 6(a)). The signal amplitude and period fluctuate with the variable speed. A zoomed-in figure within $0-0.06 \mathrm{~s}$ is shown in the middle are of Figs. 6(a) and 6(b). The waveform resembles a sinusoid, but the distortion and noise interference are clearly captured.

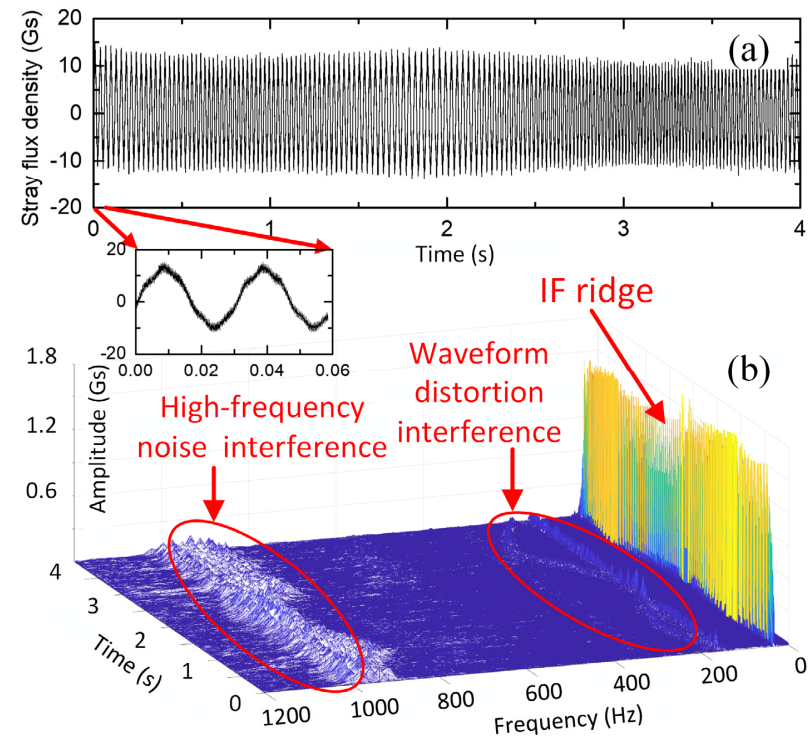

Fig. 6. (a) Magnetic signal acquired at variable speed condition and (b) time-frequency representation of the magnetic signal. 
$>$ IEEE Transactions on Energy Conversion<

The time-frequency representation is displayed in Fig. 6(b), where the yellow part on the right side depicts the dominant IF. Given that the BLDC motor is fed with trapezoidal currents, the waveform distortion induced by high-order harmonics is observed at around $200 \mathrm{~Hz}$. The high-frequency noise interference is obvious around $1000 \mathrm{~Hz}$. If the rotation angle is directly measured from the stray magnetic signal, the accuracy is affected as the interference impairs the signal.

To improve the measurement accuracy, the magnetic signal is processed using the proposed method. To reduce the computation time, the magnetic signal is downsampled with a factor of 10, and then analyzed by the WST method. The detailed parameters of the WST are configured as (using MATLAB code format): discrete scale coefficients $a_{j}=$ $2^{\wedge}(1 / 32)^{\wedge}(1: 416)$ and shift coefficients $b_{n}=(1: 20480) / 5120$ (corresponding to Eq. (7)); the hard threshold $\lambda$ for smoothing is set as $10^{\wedge}(-8)$. The IF is extracted from the time-frequency representation (Fig. 7(a)). The fluctuating IF curve indicates that the motor speed changes with time. The rotation signal component is constructed from the extracted IF (Fig. 7(b)). The length of the reconstructed signal has been extended back to 204,800 points by using numerical interpolation.

The zoomed-in figures of the original and reconstructed stray magnetic signals in Fig. 7(c) show that the latter is reasonably sinusoidal and almost noise-free. Subsequently, Hilbert transform is applied to the reconstructed signal $S_{r}[n]$ and a complex signal $H S_{r}[n]$ is obtained. The real and image parts of $H S_{r}[n]$ are plotted in Figs. 7(d) and 7(e), respectively. It can be found that the real part of $H S_{r}[n]$ is the same with the original signal $S_{r}[n]$. The accumulative rotation angle curve is derived from the signal $H S_{r}[n]$ to resample the vibration signal.

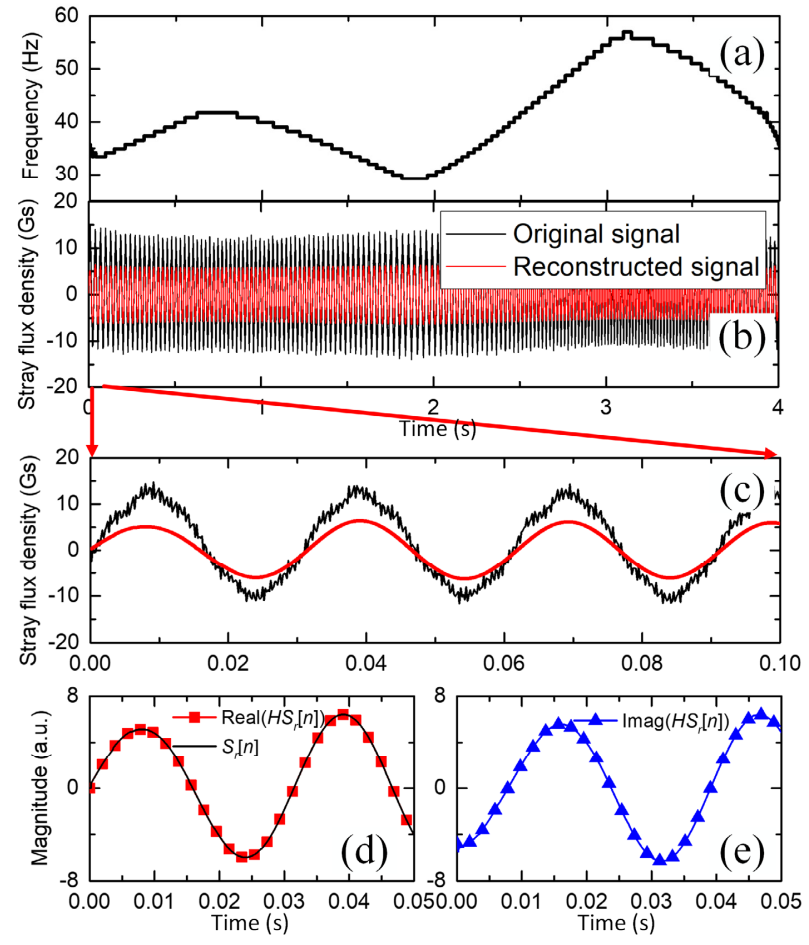

Fig. 7. (a) Extracted IF of the magnetic signal, (b) original magnetic signal and reconstructed rotation signal component, (c) zoomed-in version of (b), (d) and (e) the real and image parts of the signal $H S_{r}[n]$.
The vibration signal is analyzed for motor bearing fault diagnosis. Fig. 8 shows the vibration signal that is synchronously acquired with the magnetic signal and its envelope spectrum. The signal amplitude fluctuates with the motor speed, and the fault-induced impulse train appears in the signal waveform. After signal demodulation, the energy of the impulses transfers from the high-frequency region to the low-frequency one (Fig. 8(b)). However, the FCF changes with the motor rotation speed, and the spectral line expands and smears along with the frequency-axis. The ranges of the rotation frequency and FCF are $14-29 \mathrm{~Hz}$ and $50.54-104.69 \mathrm{~Hz}$, respectively. The magnitude of the center frequency $(77.62 \mathrm{~Hz})$ is $0.03 \mathrm{~g}$ as shown in Fig. 8(b). In practice, the range of FCF is uncertain when the rotation speed and the bearing fault type are not known before. Hence, the smearing frequency hinders the determination of the bearing fault type in the spectrum.
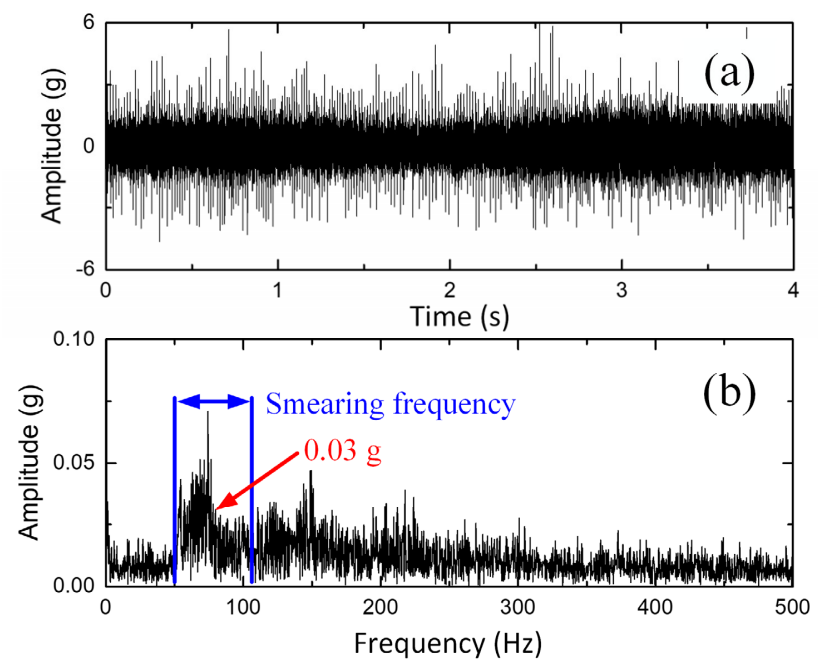

Fig. 8. (a) Synchronous sampled vibration signal and (b) its envelope spectrum.

To address this issue, an order analysis is performed on the time-domain vibration signal. The signal $V i b[n]$ is resampled on the angular domain in accordance with the instants of the rotation angle signal $R a_{m}[n]$. The resampling function in Eq. (14) interpolates $V i b[n]$ linearly onto a vector of uniformly spaced instants with the same endpoints and number of samples as $R a_{m}[n]$. More details can be referred to [28]. The resampled signal $R V i b[n]$ and its demodulated signal $\operatorname{Dem}[n]$ are shown in Figs. 9(a) and 9(b), respectively. The spectrum of $\operatorname{Dem}[n]$ is calculated and displayed in Fig. 9(c). It can be seen that the fault indicator $\mathrm{FCO}_{O}$ and its multiple harmonics are clearly displayed in the spectrum. By performing order analysis, the influence of speed variation is eliminated, and the spectral line becomes a single line with a narrow range. As confirmed by the theoretical $\mathrm{FCO}_{O}$ value in Table II, the motor bearing is subjected to an outer raceway fault. As a comparison, the magnitude of $\mathrm{FCO}_{O}$ has been improved to $0.27 \mathrm{~g}$, which is 9 times of that of the center frequency in Fig. 8(b). This result indicates that the signal resampling procedure eliminates the frequency smearing phenomenon, thereby benefiting bearing fault identification. 
>IEEE Transactions on Energy Conversion<
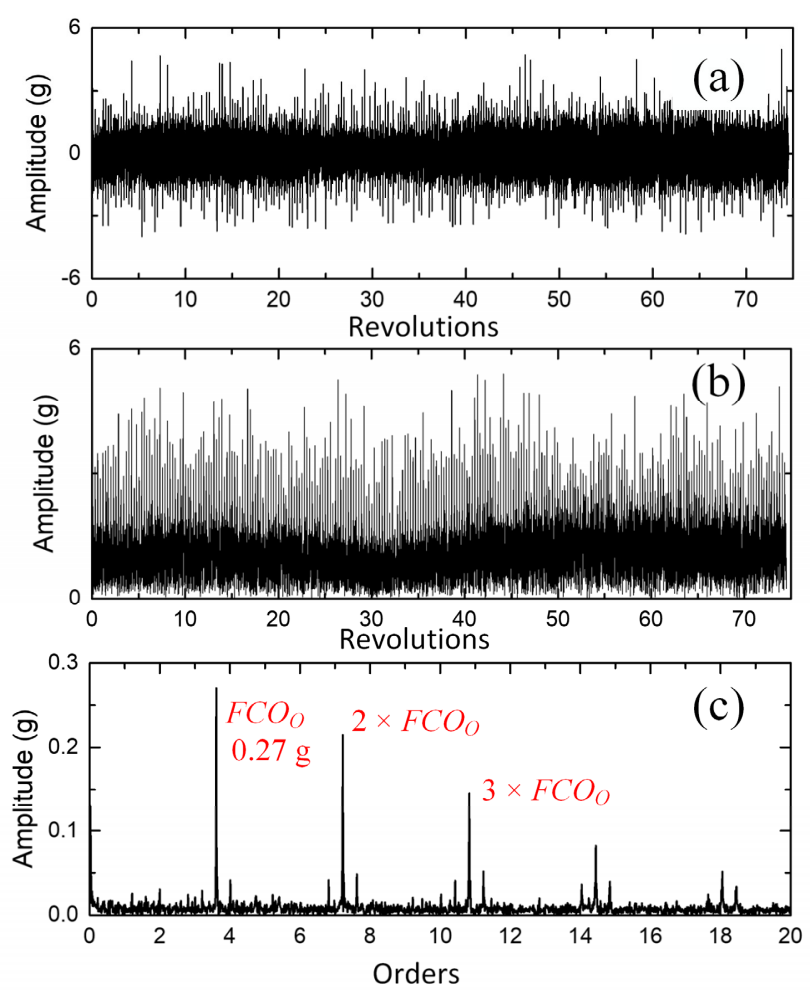

Fig. 9. (a) Resampled vibration signal based on Hall signal and (b) its demodulated signal, and (c) the spectrum of the demodulated signal.

\section{B. Diagnosis of Bearing Inner Raceway Fault with Variable Load: A Comparison Study}

To further validate the robustness of the proposed method, a bearing with inner raceway fault is tested. In this experiment, the DC electrical load is set to a constant value of $10 \Omega$. The voltage output of the generator varies with the rotation speed of the driving motor. Hence, the consumed power of the electrical load varies with time, i.e., the driving motor works at variable load conditions.
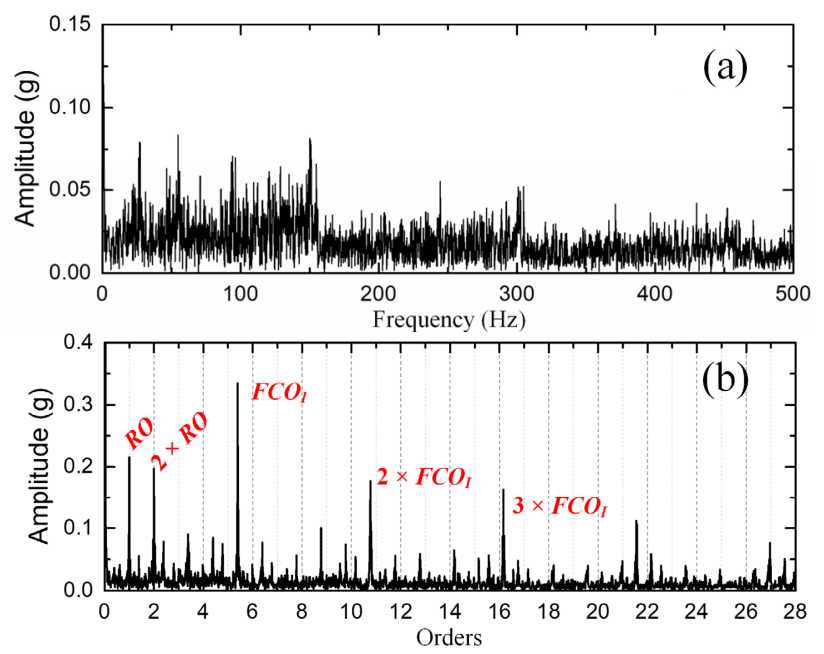

Fig. 10. (a) Envelope spectrum of the vibration signal with inner raceway fault, and (b) envelope order spectrum obtained by using the proposed method.

The envelope spectrum of the vibration signal and the envelope order spectrum processed by using the proposed method are shown in Figs. 10(a) and 10(b), respectively. The frequency bins in the the spectrum in Fig. 10(a) distribute in disorder, and no useful information can be obtained for fault identification. The vibration signal is resampled according to the rotation angle curve calculated from the Hall signal by using the proposed method. It can be seen that the rotation order (RO) and its second harmonic, and the $F C O_{I}$ and its second to fifth harmonics are clearly illustrated in Fig. 10(b). These results indicate that an inner raceway fault exists in the test bearing, which is agree with the pre-set fault type. This case study validates the robustness of the proposed method in variable load conditions.

In addition, the performance of bearing fault diagnosis is highly related to the accuracy of the estimated rotation angle. Hence, the accuracies of the estimated angles from different sensors are compared. The angles estimated from the raw Hall signal, raw current signal, and the proposed method are compared with that from the encoder signal. Partial of these angle curves are shown in Fig. 11. It can be seen that the angle curve of the proposed method is close to that of the encoder, whereas obvious deviations appear among the curves of raw Hall signal, raw current signal, and encoder signal. In particular, the angle curve is a quasi-horizontal line from around $0.490 \mathrm{~s}$ to $0.494 \mathrm{~s}$. To better illustrate such a phenomenon, the ideal phase current waveform and the measured rotor angle from current signal in six-step commutation are plotted in Fig. 12. As the motor is driven by a trapezoidal wave, the current in the energized cycle (for instance, $0-2 T_{E} / 6, T_{E}$ is a full commutation cycle) is a horizontal line. Hence, it does not yield any angle increment information. The angle increment is only obtained at the falling or rising edge of the current as shown in Figs. 12(a) and 12(b). In sum, the rotor angle curve estimated from the motor current is a discontinuous stepped curve. The comparative results indicate the high accuracy of the proposed method in angle measurement.

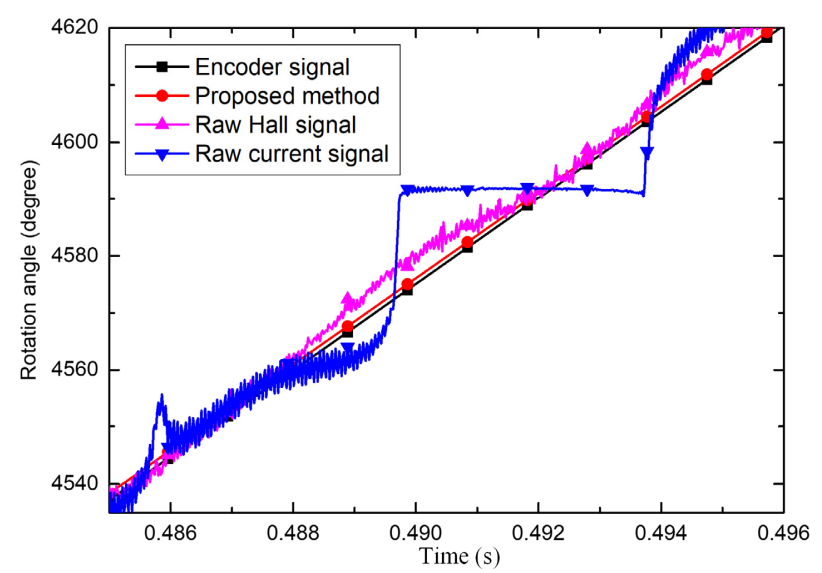

Fig. 11. Comparison of the rotation angle measurement results obtained from encoder sensor, current signal, and Hall signals with and without processing. 
>IEEE Transactions on Energy Conversion $<$

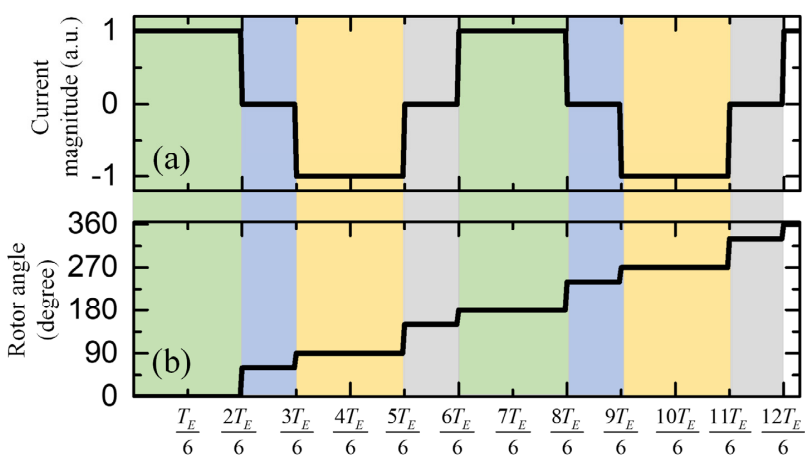

Fig. 12. BLDC motor with 2 pole pairs under six-step commutation: (a) ideal phase current waveform and (b) measured rotor angle from current signal.

The root mean square error (RMSE) between the measured angle and the referenced angle for different methods are calculated as shown in the second column in Table III. It can be found that the proposed method has the minimal RMSE value as compared with other two methods. Besides, the procedure of order analysis makes the dispersed energy of the smearing frequency components astringe to a narrow bandwidth as shown in Fig. 10. Hence, a higher $F C O_{I}$ magnitude signifies a higher resampling accuracy in the order analysis. The indexes for different methods are calculated as shown in the third column in Table III. The maximal $F C O_{I}$ magnitude is obtained when the encoder signal is used for signal resampling, because the encoder generates the highest angle measurement accuracy. The magnitude of the proposed method is close to that of the encoder, which indicates that the Hall signal processed by using the proposed method has a relative high accuracy.

As shown in Figs. 9(b) and 10(b), bearing fault type can be determined by the FCO and its harmonics. For a given vibration signal, the harmonic magnitude can reflect the accuracy of a fault diagnosis method. Here, the sum of frequency magnitudes from $1 \times F C O_{I}$ to $5 \times F C O_{I}$ are calculated and shown in the fourth column in Table III. The result of the proposed method is higher than that of the Hall and current signals without processing.

TABLE III

COMPARISON OF DIFFERENT METHODS

\begin{tabular}{ccccc}
\hline \hline Method & $\begin{array}{c}\text { Angle } \\
\text { RMSE }\left({ }^{\circ}\right)\end{array}$ & $\begin{array}{c}F C O_{I} \\
\text { Mag. }(\mathrm{g})^{*}\end{array}$ & $\begin{array}{c}\text { Harmonics } \\
\text { Mag. }(\mathrm{g})\end{array}$ & $\begin{array}{c}\text { Comp. } \\
\text { time (s) }\end{array}$ \\
\hline Encoder (base line) & - & 0.3451 & 1.5443 & 0.2301 \\
Proposed & 0.4323 & 0.3343 & 0.9626 & 7.3348 \\
Raw Hall & 2.1967 & 0.3150 & 0.7240 & 0.2599 \\
Raw current & 6.3791 & 0.2829 & 0.3759 & 0.2271 \\
\hline \hline *
\end{tabular}

Finally, the computation time is evaluated to make the proposed method more explicit in practical applications. The platform for computation is configured as: Quad-Core $1.8 \mathrm{GHz}$ CPU, 8 GB Memory, WIN10 OS, and MATLAB 2019b. Because extra time-frequency analysis is conducted, the computation time of the proposed method is larger than that of other methods. Nevertheless, considering that the monitoring period of the bearing is generally multiple minutes to hours, the computation time $(\sim 7 \mathrm{~s})$ is far less than the monitoring period. In other words, online bearing condition monitoring and fault diagnosis can be realized by using the proposed method. With the rapid development of the hardware platform, the computation time can be further decreased in the future. The advantage of the proposed method in rotation angle measurement from stray magnetic signals is confirmed by the comparative analysis.

\section{Method Robustness Evaluation}

A quantitative analysis is conducted to evaluate the robustness of the proposed method. A coordinate system is constructed on the motor side (Fig. 13), and the Hall sensor is attached to the center of the side face at $(5 \mathrm{~cm}, 2 \mathrm{~cm})$. As shown in Fig. 13, the coordinates of the Hall sensor change within the grid with a step of $1 \mathrm{~cm}$. The range of the coordinates in the $x$-axis is within $0-10 \mathrm{~cm}$, whereas that in the $y$-axis is within $0-4 \mathrm{~cm}$. The total number of test locations is 55 .

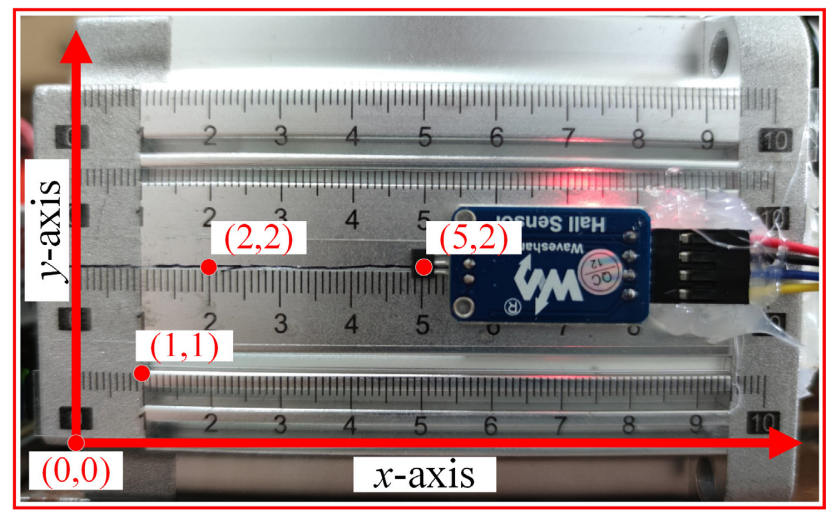

Fig. 13. Hall sensor locations for sensing the stray flux density.

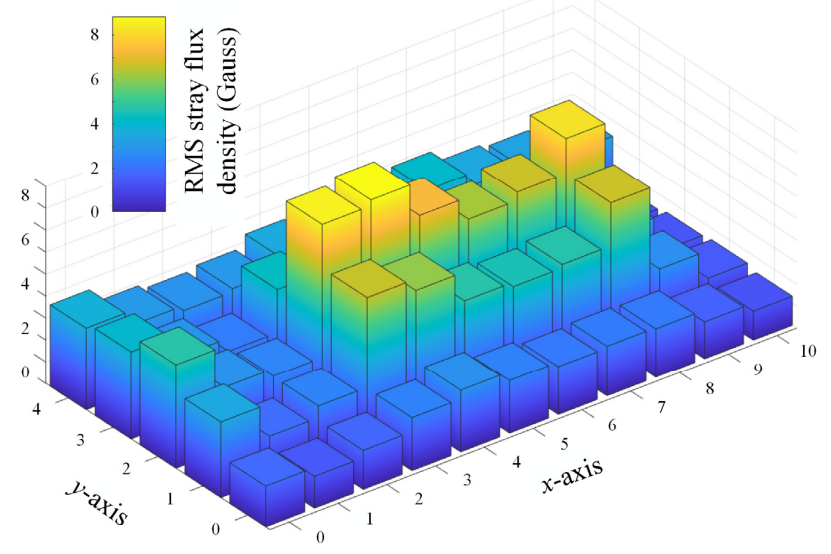

Fig. 14. RMS values of the stray flux density at different locations.

The BLDC motor is set to rotate at a constant speed. The root mean square (RMS) value of the stray flux density is measured and plotted in Fig. 14. Large RMS values are observed at the central area of the side face. RMS decreases in both axes as the distance of the Hall sensor from the central area increases. This phenomenon can be explained by two reason. First, the lengths of the rotor mounted with magnets and stator winding in the $x$-axis are less than that of the motor housing. Therefore, the leakage flux, which is a part of the air-gap flux between the stator and the rotor, will decrease as the Hall sensor deviates 
$>$ IEEE Transactions on Energy Conversion<

from the central area. Second, because the stator yoke is circular and the motor housing is flat in the $y$-axis, the vertical displacement between the air gap and the sensor decreases when the Hall sensor deviates from the center. From the measurement perspective, a high sensor output magnitude will improve the signal-to-noise ratio (SNR) if the background noise remains at a certain level. High output SNR is beneficial for further signal analysis. Therefore, the Hall sensor should be placed at the central area rather than in the edge of the side face of the motor housing.

To further assess the rotation angle measurement accuracy, the RMSE between the angles of the Hall and encoder signals is calculated. The RMSE values at different sensor locations are shown in Fig. 15. A lower RMSE value indicates a higher rotation angle measurement accuracy and vice versa. The findings reveal that high accuracy values are generated in the central area. This phenomenon is consistent with the results of the RMS experiment in Fig. 14, that is, the magnetic signal obtains high output magnitude and high SNR when the Hall sensor is placed in the central area. As a result, the accuracy improves. The minimum RMSE value is observed at $0.37^{\circ}$ at ( 4 $\mathrm{cm}, 2 \mathrm{~cm})$. The resolution of the encoder is $0.36^{\circ}$ degree because the rising and falling edges of the encoder pulses are used to calculate the rotation angle in this study. The results indicate that the proposed method achieves high accuracy in rotation angle measurement. The RMSE values of 21 locations are lower than $1^{\circ}$, which means that the proposed method is robust when the sensor is placed at different locations. Installing the Hall sensor at the central area will yield satisfactory results of motor rotation angle measurement and fault diagnosis.

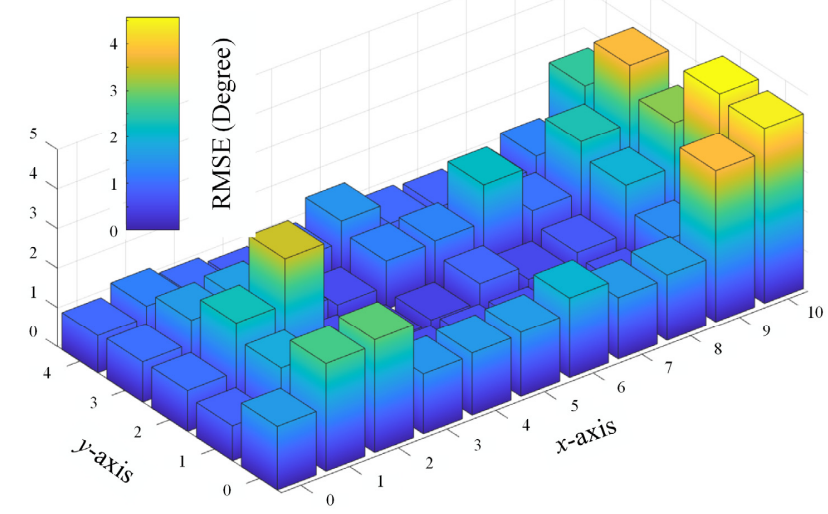

Fig. 15. RMSE values of rotation angle measurements at different locations.

\section{DISCUSSIONS}

Stray flux has been widely investigated for measurement of motor parameters and conditions including current, power, mechanical and electrical faults. In this study, the stray flux signal is used for rotation angle measurement of motor shaft. As indicated from the above experimental results, if the encoder signal is not available or wiring cables is not convenient, the proposed method based on stray flux measurements can provide an alternative solution for realizing fault diagnosis under varying speed conditions. According to this principle, a hand-held instrument can be designed for noninvasive, nondestructive, and in situ motor bearing fault diagnosis as illustrated in Fig. 16. Such an instrument provides much more conveniences as it doesn't need to access to the motor drive and control systems which could have potential hazards. Indeed, the measurement principle can be extended to other types of synchronous motors, and also asynchronous motors if the slip rate is known.

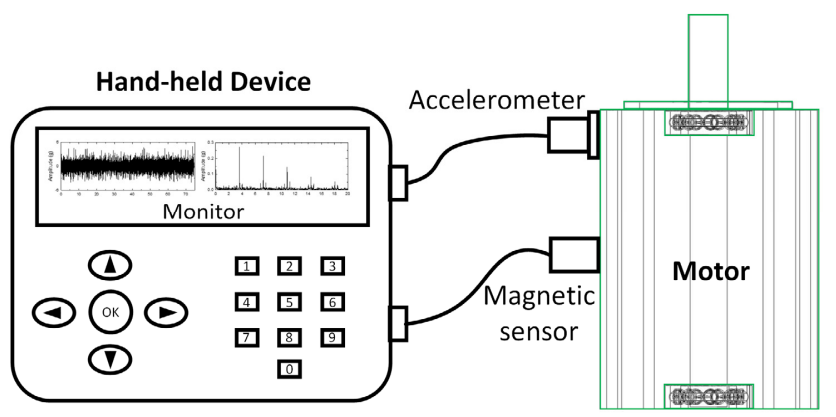

Fig. 16. Illustration of hand-held device for noninvasive, nondestructive, and in situ motor bearing condition monitoring and fault diagnosis.

\section{CONCLUSIONS}

This paper has presented a novel method based on stray magnetic signal analysis for BLDC motor rotation angle measurement. The principle and performance of the proposed approach were comprehensively discussed, and the potential applications in motor fault diagnosis under varying speed conditions were validated through experiments. The robustness of the proposed method was also evaluated through a quantitative analysis. The experimental results indicated that the accuracy of rotation angle measurement obtained using the external Hall sensor was similar to that obtained using conventional optical encoders. As the stray magnetic signal can be acquired from the motor housing by using a low-cost Hall sensor, the proposed method provides an efficient and feasible alternative to motor rotation angle measurements. It is nonintrusive and eliminates the use of long cables and encoders in motor drives. Moreover, the proposed approach can be extended to the diagnosis of other electrical and drive faults of motors under varying speed conditions. If implemented with an IoT or a hand-held device, it can further improve the reliability of sensorless motor drive systems in industrial automation. This will be developed in the future work in order to meet Industry 4.0 requirements in industrial automation applications.

\section{REFERENCES}

[1] K. Al Jaafan and H. A. Toliyat, "Performance Analysis of Synchronous Generators Under Stator Windings Ground Faults Near the Star Point-Experimental Verification," IEEE Trans. Energy Convers., vol. 35, pp. 1402-1410, Sep. 2020.

[2] S. Afrandideh, M. E. Milasi, F. Haghjoo, and S. M. A. Cruz, "Turn to Turn Fault Detection, Discrimination, and Faulty Region Identification in the Stator and Rotor Windings of Synchronous Machines Based on the Rotational Magnetic Field Distortion," IEEE Trans. Energy Convers., vol. 35, pp. 292-301, Mar. 2020 
$>$ IEEE Transactions on Energy Conversion $<$

[3] T. Goktas, M. Zafarani, and B. Akin, "Discernment of Broken Magnet and Static Eccentricity Faults in Permanent Magnet Synchronous Motors," IEEE Trans. Energy Convers., vol. 31, pp. 585-594, Jun. 2016.

[4] R. Z. Haddad, "Detection and Identification of Rotor Faults in Axial Flux Permanent Magnet Synchronous Motors Due to Manufacturing and Assembly Imperfections," IEEE Trans. Energy Convers., vol. 35, pp. 174-183, Mar. 2020.

[5] R. Puche-Panadero, J. Martinez-Roman, A. Sapena-Bano, and J. Burriel-Valencia, "Diagnosis of Rotor Asymmetries Faults in Induction Machines Using the Rectified Stator Current," IEEE Trans. Energy Convers., vol. 35, pp. 213-221, Mar. 2020.

[6] S. Nandi, H. A. Toliyat, and X. D. Li, "Condition monitoring and fault diagnosis of electrical motors - A review," IEEE Trans. Energy Convers., vol. 20, pp. 719-729, Dec. 2005.

[7] J. Zhu, N. Chen, and W. W. Peng, "Estimation of Bearing Remaining Useful Life Based on Multiscale Convolutional Neural Network," IEEE Trans. Ind. Electron., vol. 66, pp. 3208-3216, Apr. 2019.

[8] S. Lu, R. Yan, Y. Liu, and Q. Wang, "Tacholess Speed Estimation in Order Tracking: A Review with Application to Rotating Machine Fault Diagnosis," IEEE Trans. Instrum. Meas., vol. 68, pp. 2315-2332, Jul. 2019.

[9] M. Zhao, J. Lin, X. Q. Xu, and Y. Lei, "Tacholess Envelope Order Analysis and Its Application to Fault Detection of Rolling Element Bearings with Varying Speeds," Sensors, vol. 13, pp. 10856-10875, Aug. 2013.

[10] S. Tsotoulidis and A. N. Safacas, "Deployment of an Adaptable Sensorless Commutation Technique on BLDC Motor Drives Exploiting Zero Sequence Voltage," IEEE Trans. Ind. Electron., vol. 62, pp. 877-886, Feb. 2015.

[11] Y. Hu, X. T. Tu, F. C. Li, H. G. Li, and G. Meng, "An adaptive and tacholess order analysis method based on enhanced empirical wavelet transform for fault detection of bearings with varying speeds," J. Sound Vib. vol. 409, pp. 241-255, Nov. 2017.

[12] J. F. Zhong, S. C. Zhong, Q. K. Zhang, Z. K. Peng, S. L. Liu, and Y. J. Yu, "Vision-Based Measurement System for Instantaneous Rotational Speed Monitoring Using Linearly Varying-Density Fringe Pattern," IEEE Trans. Instrum. Meas., vol. 67, pp. 1434-1445, Jun. 2018.

[13] J. Wang, Y. Peng, and W. Qiao, "Current-Aided Order Tracking of Vibration Signals for Bearing Fault Diagnosis of Direct-Drive Wind Turbines," IEEE Trans. Ind. Electron., vol. 63, pp. 6336-6346, Oct. 2016.

[14] T. Wang, M. Liang, J. Li, and W. Cheng, "Rolling element bearing fault diagnosis via fault characteristic order (FCO) analysis," Mech. Syst. Signal Process., vol. 45, pp. 139-153, 2014.

[15] J. Zhong, S. Zhong, Q. Zhang, N. Mafia, Y. Shen, S. Liu, et al., "Vision-based system for simultaneous monitoring of shaft rotational speed and axial vibration using non-projection composite fringe pattern," Mech. Syst. Signal Process., vol. 120, pp. 765-776, Apr. 2019.

[16] Z. Liu, G. Y. Tian, W. P. Cao, X. W. Dai, B. Shaw, and R. Lambert, "Non-invasive load monitoring of induction motor drives using magnetic flux sensors," IET Power Electron., vol. 10, pp. 189-195, Feb. 2017.

[17] L. Frosini, C. Harlisca, and L. Szabo, "Induction Machine Bearing Fault Detection by Means of Statistical Processing of the Stray Flux Measurement," IEEE Trans. Ind. Electron., vol. 62, pp. 1846-1854, Mar. 2015

[18] J. W. Shao, D. Nolan, M. Teissier, and D. Swanson, "A novel microcontroller-based sensorless brushless DC (BLDC) motor drive for automotive fuel pumps," IEEE Trans. Ind. Appl., vol. 39, pp. 1734-1740, Nov.-Dec. 2003.

[19] X. Y. Liu, C. H. Liu, and P. W. T. Pong, "Velocity Measurement Technique for Permanent Magnet Synchronous Motors Through External Stray Magnetic Field Sensing," IEEE Sens. J., vol. 18, pp. 4013-4021, May 2018

[20] A. A. Adam, K. Gulez, and S. Koroglu, "Stray magnetic field distributed around a PMSM," Turkish J. Electr. Eng. Comput. Sci., vol. 19, pp. 119-131, 2011.

[21] T. Goktas, M. Zafarani, K. W. Lee, B. Akin, and T. Sculley, "Comprehensive Analysis of Magnet Defect Fault Monitoring Through Leakage Flux," IEEE Trans. Magn., vol. 53, Apr. 2017.

[22] R. H. Qu and T. A. Lipo, "Analysis and modeling of air-gap and zigzag leakage fluxes in a surface-mounted permanent-magnet machine," IEEE Trans. Ind. Appl., vol. 40, pp. 121-127, Jan.-Feb. 2004.

[23] S. Q. Chen, X. J. Dong, Z. K. Peng, W. M. Zhang, and G. Meng, "Nonlinear Chirp Mode Decomposition: A Variational Method," IEEE Trans. Signal Process., vol. 65, pp. 6024-6037, Nov. 2017.

[24] Z. P. Feng, X. W. Chen, and T. Y. Wang, "Time-varying demodulation analysis for rolling bearing fault diagnosis under variable speed conditions," J. Sound Vib., vol. 400, pp. 71-85, Jul. 2017.
[25] I. Daubechies, J. F. Lu, and H. T. Wu, "Synchrosqueezed wavelet transforms: An empirical mode decomposition-like tool," Appl. Comput. Harmon. A., vol. 30, pp. 243-261, Mar. 2011.

[26] S. Meignen, T. Oberlin, and S. McLaughlin, "A New Algorithm for Multicomponent Signals Analysis Based on SynchroSqueezing: With an Application to Signal Sampling and Denoising," IEEE Trans. Signal Process., vol. 60, pp. 5787-5798, Nov. 2012.

[27] G. Thakur, E. Brevdo, N. S. Fuckar, and H. T. Wu, "The Synchrosqueezing algorithm for time-varying spectral analysis: Robustness properties and new paleoclimate applications," Signal Process., vol. 93, pp. 1079-1094, May 2013

[28] Introduction of MATLAB function of resample (accessed on Jan. 25 , 2021). Available: https://www.mathworks.com/help/signal/ref/resample.html?s tid=srchtitle

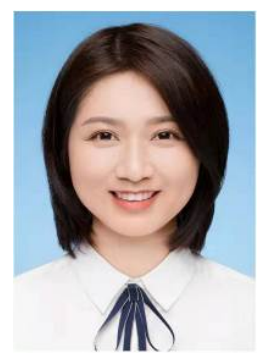

Xiaoxian Wang (Member, IEEE) received the B.S. degree in mechanical engineering from Shandong University of Science and Technology, Qingdao, China, in 2010, the M.S. degree in mechanical engineering in 2013 from the University of Science and Technology of China, Hefei, China.

She is currently an Engineer with the College of Electronics and Information Engineering, Anhui University, Hefei, China, and also a visiting scholar with the Traction Power State Key Laboratory, Southwest Jiaotong University, Chengdu, China. She is currently pursuing the Ph.D. degree with the Department of Precision Machinery and Precision Instrumentation, University of Science and Technology of China, Hefei, China. Her current research interests include innovative design and intelligent maintenance of electromechanical system.

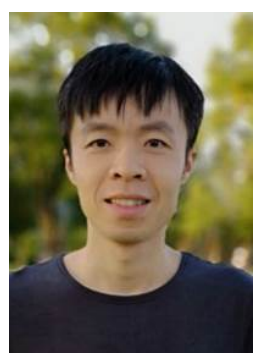

Siliang Lu (Senior Member, IEEE) received the B.S. and Ph.D. degrees in mechanical engineering from the University of Science and Technology of China, Hefei, China, in 2010 and 2015, respectively.

$\mathrm{He}$ is currently an Associate Professor with the College of Electrical Engineering and Automation, Anhui University, Hefei, China, and also a visiting scholar with the Traction Power State Key Laboratory, Southwest Jiaotong University, Chengdu. His current research interests include machinery condition-based monitoring and fault diagnosis, signal processing, IoT and edge computing, and robotics.

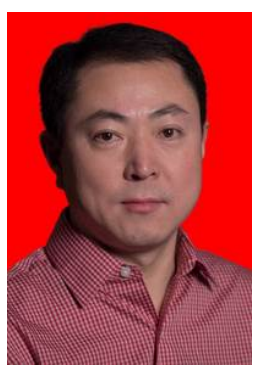

Wenping Cao (Senior Member, IEEE) received the B.Eng. degree in electrical engineering from Beijing Jiaotong University, Beijing, China, in 1991, and the $\mathrm{Ph}$.D. degree in electrical machines and drives from the University of Nottingham, Nottingham, U.K., in 2004.

He was a Chair Professor of Electrical Power Engineering and the Head of the Power Electronics, Machines, and Power System Group, Aston University, Birmingham, U.K. He is currently a Distinguished Professor at Anhui University, China. He serves as an Editor for the IEEE Transactions on Power Electronics, IEEE Transactions on Industry Applications, IEEE Industry Applications Magazine, IET Power Electronics, and Electric Power Components and Systems.

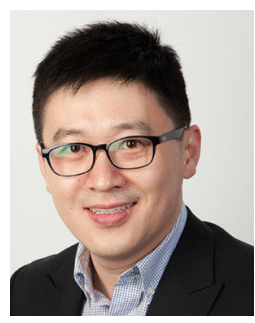

Min Xia (Member, IEEE) received the B.S. degree from Southeast University, Nanjing, China, in 2009, the M.S. degree from the University of Science and Technology of China, Hefei, China, in 2012, and the Ph.D. degree from the University of British Columbia, Vancouver, Canada, in 2017.

He is currently a Lecturer with the Department of Engineering, Lancaster University, Lancaster, U.K. His research interests include smart manufacturing, machine diagnosis and prognosis, deep neutral networks, autonomous vehicle, and sensor fusion. 
>IEEE Transactions on Energy Conversion<

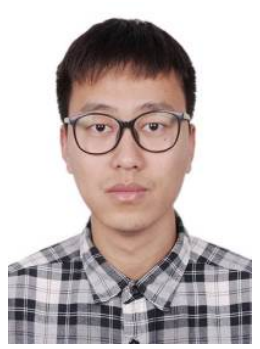

Kang Chen received the B.S. degree in mechanical engineering from West Anhui University, in 2018.

$\mathrm{He}$ is currently pursuing the master degree with the College of Electrical Engineering and Automation, Anhui University, Hefei, China.

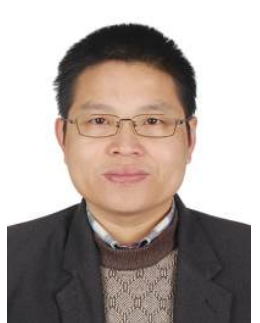

Jianming Ding received the Ph.D. degree in mechanical engineering from the Southwest Jiaotong University, Chengdu, China, in 2011.

$\mathrm{He}$ is currently an Associate Researcher with the Traction Power State Key Laboratory, Southwest Jiaotong University, Chengdu, China. He was a visiting scholar with The University of Western Australia, Australia from 2016 to 2017. His research interests include fault diagnosis of vehicles, signal processing, experimental test rig development.

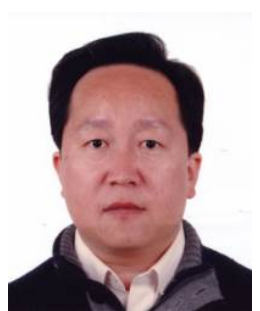

Shiwu Zhang (Member, IEEE) received the BS degree (1997) in mechanical and electrical engineering from University of Science and Technology of China (USTC), and the PhD degree (2003) in precision instrumentation and precision machinery from USTC, Hefei, China.

$\mathrm{He}$ is currently a professor in the Department of Precision Machinery and Precision Instrumentation, USTC. He is the named author of over 100 papers in different peer-reviewed journals and conferences. His current research interests include precision instrumentation, smart materials and their applications in bio-inspired robots, and soft robots. 